\section{Vorhersagewert, negativer}

R.-D. Hilgers ${ }^{1}$, N. Heussen ${ }^{1}$ und S. Stanzel ${ }^{2}$

${ }^{1}$ Institut für Medizinische Statistik, Universitätsklinikum der RWTH Aachen, Aachen, Deutschland

${ }^{2}$ DKFZ Heidelberg, Heidelberg, Deutschland

Synonym(e) Prädiktiver Wert des negativen Testresultats

Englischer Begriff negativ predictive value

Definition Der negative Vorhersagewert oder prädiktive Wert des negativen Testresultats ist definiert als die (bedingte) Wahrscheinlichkeit, gesund zu sein, falls ein negatives Testergebnis vorliegt.

Beschreibung Der negative Vorhersagewert wird geschätzt durch den Quotienten aus der Zahl der Gesunden mit negativem Test und der Gesamtzahl der testnegativen Fälle (d. h. durch den Quotienten d / $(c+d)$; Bezeichnungen s. Tabelle im Eintrag $>$ Vierfeldertafel).

Der prädiktive Wert des negativen Testresultats lässt sich mithilfe des Satzes von Bayes ( $\triangleright$ Bayes, Satz von) aus der Sensitivität (Se; $>$ Sensitivität, diagnostische), der Spezifität $(\mathrm{Sp} ; \triangleright$ Spezifität, diagnostische) und der \ Prävalenz (prev) berechnen:

$$
\mathrm{PV}^{-}=\frac{\mathrm{Sp} \times(1-\text { prev })}{\mathrm{Sp} \times(1-\text { prev })+(1-\mathrm{Se}) \times \text { prev }}
$$

Für den Zusammenhang zwischen dem negativen Vorhersagewert der Prävalenz und der Sensitivität gilt, dass der Vorhersagewert sinkt, wenn die Prävalenz steigt bzw. dass der negative Vorhersagewert bei hoher Sensitivität hoch ist.

\section{Literatur}

Hilgers R-D, Bauer P, Scheiber V (2002) Einführung in die Medizinische Statistik. Springer, Berlin/Heidelberg/New York 